\title{
Complement-independent Ab-induced peroxide lysis of platelets requires 12-lipoxygenase and a platelet NADPH oxidase pathway
}

\author{
Michael Nardi, ${ }^{1}$ Steven J. Feinmark, ${ }^{2}$ Liang Hu, ${ }^{3}$ Zongdong $\mathrm{Li}^{3}{ }^{3}$ and Simon Karpatkin ${ }^{3}$ \\ 1Department of Pediatrics, New York University School of Medicine, New York, New York, USA. \\ 2Department of Pharmacology, Center for Molecular Therapeutics, Columbia College of Physician and Surgeons, New York, New York, USA. \\ ${ }^{3}$ Department of Medicine, New York University School of Medicine, New York, New York, USA.
}

\begin{abstract}
Antiplatelet GPIIIa49-66 Ab of HIV-related thrombocytopenic patients induces thrombocytopenia and platelet fragmentation by the generation of peroxide and other reactive oxygen species (ROS). Here we report the presence of a functional platelet NADPH oxidase pathway that requires activation by the platelet 12-lipoxygenase (12-LO) pathway to fragment platelets. A new Ab-mediated mechanism is described in which the platelet 12-LO product, 12(S)-HETE activates the NADPH oxidase pathway to generate ROS.
\end{abstract}

\section{Introduction}

Patients with HIV-1 infection commonly develop immunologic thrombocytopenia (HIV-1-ITP) (1). Platelet survival studies indicate that early-onset HIV-1-ITP is often due to increased peripheral destruction of platelets, whereas patients with AIDS are more likely to develop decreased platelet production (2). Early-onset HIV-1-ITP is clinically indistinguishable from classic idiopathic autoimmune thrombocytopenia (AITP) and responsive to the same therapeutic agents used to treat classic AITP (1, 3-6). HIV-1-ITP is different from classic AITP, however, with respect to markedly elevated platelet-associated IgG, IgM, C3, C4, and the presence of circulating immune complexes (CICs) composed of the same $(3,4)$.

Past studies have revealed that these complexes also contain antiplatelet integrin GPIIIa ( $\beta 3$ ) Ab and its anti-idiotype blocking $\mathrm{Ab}(7,8)$. Affinity purification of the $\mathrm{Ab}$ and epitope mapping of GPIIIa have revealed a high-affinity IgG1 Ab against GPIIIa residues 49-66 $(7,9)$. The presence of this Ab correlates inversely with platelet count $(r=-0.71)$ and induces severe thrombocytopenia when introduced into mice (9) (mouse GPIIIa has $83 \%$ homology with human GPIIIa and macrophages with Fc receptors for human IgG1). Passively induced murine thrombocytopenia can be prevented or reversed with GPIIIa49-66 peptide (9) as well as patient anti-idiotype blocking $\mathrm{Ab}(8)$.

Circulating immune complexes from early-onset HIV-1-ITP patients contain platelet fragments (10). We have also demon-

Nonstandard abbreviations used: autoimmune thrombocytopenia (AITP); cinnamyl-3,4-dihydroxy- $\alpha$-cyanocinnamate (CDC); circulating immune complexes (CICs); cytosolic phospholipase $\mathrm{A}_{2}$ ( $\mathrm{CPLA}_{2}$ ); DCFH diacetate (DCFH-DA); dichlorodihydrofluorescein (DCFH); diphenylene iodonium (DPI); 5,8,11-eicosatriynoic acid (ETI); extracellular signal-regulated kinase (ERK); flavin adenine dinucleotide (FAD); immunologic thrombocytopenia (HIV-1-ITP); leukotriene $\mathrm{B}_{4}\left(\mathrm{LTB}_{4}\right)$; 12-lipoxygenase (12-LO); nordihydroguaiaretic acid (NDGA); P13 kinase substrate (AKT); p38 mitogen-activated protein kinase (p38 MAPK); pertussis toxin (PTX); phagocytic oxidase (phox); reactive oxygen species (ROS)

Conflict of interest: The authors have declared that no conflict of interest exists.

Citation for this article: J. Clin. Invest. 113:973-980 (2004)

doi:10.1172/JCI200420726. strated that affinity-purified anti-GPIIIa49-66, in the absence of complement, can induce platelet fragmentation in vitro and in vivo (10). This intriguing observation led to the discovery that Abinduced platelet fragmentation was due to the generation of peroxide and other reactive oxygen species (ROS). The generation of ROS appeared to be due to the presence of an active NADPH oxidase pathway in platelets, since in vivo platelet fragmentation did not occur in mice deficient in the $\mathrm{p} 47$ phagocytic oxidase (p47phox) component of the NADPH ( 447 phox-/-) pathway and in vivo thrombocytopenia was approximately $60 \%$ less than that obtained with WT mice (10). The mechanism leading to this activation of ROS, however, was not defined nor was it clear whether the ROS activity was derived from the platelet or some other source.

The NADPH oxidase of granulocytes/macrophages is composed of five major components that coalesce onto the cell or vacuolar membrane to form an active electron donor in which superoxide, $\mathrm{O}_{2}{ }^{-}$, is generated (11). Three cytoplasmic phox components, $\mathrm{p} 47^{\mathrm{phox}}, \mathrm{p} 67^{\mathrm{phox}}$, and $\mathrm{p} 40^{\mathrm{phox}}$, translocate to the cytoplasmic surface of the membrane (12-16) in independent association with activated Rac $\mathrm{G}$ protein. Rac binds to p67phox (17), and they then bind to two membrane-localized components, gp91 phox $^{\text {and }}$ 22 2 hox , the $\alpha$ and $\beta$ subunits of the cytochrome $b$ complex $(18,19)$. This complex can bind NADPH and flavin adenine dinucleotide (FAD) $(11,20,21)$. Several enzymes are required for the system to be activated after membrane perturbation by way of various physiologic ligands (fMLP, C5a, PAF, leukotriene $\mathrm{B}_{4}\left[\mathrm{LTB}_{4}\right]$, IL-8) that bind to pertussis toxin-sensitive (PTX-sensitive) G protein-coupled receptors (22). Activation is dependent upon lipid mediators such as phosphatidic and arachidonic acids and phosphatidylinositol (23-25). The enzymes involved include PI3K, whose products form a scaffold for membrane attachment of $\mathrm{p} 40$ phox and $\mathrm{p} 47 \mathrm{phox}$ (26-28); protein kinase $\mathrm{C}$, which phosphorylates $\mathrm{p} 47 \mathrm{phox}$, enabling its translocation to the membrane (29-31); cytosolic phospholipase $\mathrm{A}_{2}$ $\left(\mathrm{CPLA}_{2}\right)$, which generates arachidonic acid from membrane phospholipids (32), serving to activate the association of $\mathrm{p} 47 \mathrm{phox}$ with p22phox (23). Extracellular signal-regulated kinase (ERK) and p38 
mitogen-activated protein kinase (p38 MAPK) are required for the phosphorylation and activation of $\mathrm{CPLA}_{2}(33-36)$. In both phagocytic and nonphagocytic cells, $\mathrm{cPLA}_{2}$ participates in the generation of leukotriene $\mathrm{B}_{4}\left(\mathrm{LTB}_{4}\right)$, which appears to be required for ROS generation and chemotaxis (37). The relationship between $\mathrm{LTB}_{4}$ and NADPH oxidase is unknown.

Here we (a) demonstrate that platelets have a functional, NADPH oxidase pathway; (b) indicate that the pathway is activated by membrane perturbation of GPIIIa49-66; and (c) describe a new ROS pathway in which 12 (S)-HETE, a product of the platelet 12-lipoxygenase (12-LO), activates NADPH oxidase following Ab ligation of GPIIIa49-66. Thus, complement-independent $\mathrm{Ab}$-induced platelet fragmentation through NADPH oxidase generation of ROS requires activation of platelet 12-LO.

\section{Results}

Absence of granulocytes in gel-filtered platelet preparations. Figure 1A demonstrates the absence of granulocytes/monocytes in a human gel-filtered platelet preparation as examined by flow cytometry with a granulocyte/macrophage-specific anti-CD11b $\mathrm{Ab}$. It could be shown that greater than $99 \%$ of the platelets were free of $\mathrm{CD} 11 \mathrm{~b}$ reactivity. This was confirmed by a phase microscopy analysis of 2,000 platelets, in which no leukocytes were visualized. Thus, contamination of platelets with granulocytes/monocytes or cross talk between both cells was ruled out.

Presence of active NADPH oxidase in murine platelets and gp91phox in buman platelets. Although our previous studies have indicated that ROS are necessary for Ab-mediated platelet fragmentation, the source of the ROS was not defined. In our previous report we demonstrated absence/impairment of anti-GPIIIa49-66-induced platelet fragmentation/thrombocytopenia in vivo with murine NADPH oxidase-deficient p47phox- $/$ - mice, but did not examine the effect of $\mathrm{Ab}$ on platelet fragmentation in vitro. Therefore, a systemic effect or granulocyte/macrophage effect was not ruled out. We therefore incubated a different NADPH oxidase-deficient platelet preparation from gp 91 phox-/- mice with antiplatelet $\mathrm{Ab}$. The

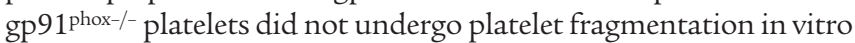
when exposed to the anti-GPIIIa49-66 Ab (percentage fragmentation in control, $6.4 \% \pm 0.06 \%$, versus patient IgG, $5.9 \% \pm 0.06 \%$ ), whereas WT mouse platelets did fragment (percentage fragmentation in control, $5.7 \% \pm 0.06 \%$, versus patient IgG, $31.8 \% \pm 5.4 \%$ ), ruling out a systemic effect.

The presence of membrane gp91 phox was also demonstrated in human gel-filtered platelets by flow cytometry with a specific $\mathrm{mAb}$ against gp91phox (Figure 1B). Platelets had significant reactivity that was similar to that seen in granulocytes.

Classical inhibitors of the NADPH oxidase pathway inhibit Ab-induced platelet fragmentation in vitro. To determine whether platelets had a functioning NADPH oxidase pathway responsive to membrane perturbation, we employed classical inhibitors of the system to determine whether or not they would inhibit Ab-induced platelet fragmentation. PTX, an inhibitor of $\mathrm{G}$ protein-coupled receptors, inhibited in vitro platelet fragmentation at $5 \mathrm{ng} / \mathrm{ml}$ (28\% fragmentation in untreated versus $5.5 \%$ after PTX and $6 \%$ after control treatment, representative of two similar experiments). Similar results were obtained with other inhibitors (Figure 2) known to interfere with this pathway. $\mathrm{IC}_{50}$ values for inhibition of several key enzymes were estimated to be PI3 kinase with LY294002 at $10 \mu \mathrm{M}$, protein kinase C with bisindolylmaleimide at $20 \mathrm{nM}$, p38 MAPK with SB203290 at $5 \mu \mathrm{M}$, and $\mathrm{PLA}_{2}$ with bromophenacyl bromide at $50 \mu \mathrm{M}$.
Ab-induced functional activation of the NADPH oxidase pathway. Platelets were incubated with $\mathrm{Ab}$ purified from control subjects or from patients with HIV-1-ITP. Patient Ab, but not control, induced activation (phosphorylation) of P13 kinase substrate (AKT) 2.5fold at 30 minutes (Figure 3A; densitometry compared with internal control). The time lag is compatible with the lag period obtained prior to platelet fragmentation. Similar activation of ERK was also noted of 2.1- and 2.3-fold at 15 and 30 minutes, respectively (Figure $3 \mathrm{~B}$ ). Figure $3 \mathrm{C}$ demonstrates $\mathrm{Ab}$-induced translocation of $\mathrm{p}^{4} 7^{\mathrm{phox}}$, $\mathrm{p} 67_{\mathrm{phox}}$, and $\mathrm{CPLA}_{2}$ to the membrane, as has been noted with granulocyte activation of NADPH oxidase.

Role of platelet lipoxygenase in Ab-induced platelet fragmentation. Because the lipoxygenase product, $\mathrm{LTB}_{4}$, has been shown to induce ROS in nonphagocytic cells, we elected to examine platelet lipoxygenase products for a possible role in $\mathrm{Ab}$-induced platelet fragmentation. Platelets have a unique isoform of the $12-\mathrm{LO}$, distinct in structure and kinetics from the enzyme found in granulocytes. We tested the ability of several distinct inhibitors of the platelet 12-LO to block Ab-induced platelet fragmentation. Dose-response data for inhibition by 5,8,11-eicosatriynoic acid (ETI), nordihydroguaiaretic acid (NDGA), and the selective 12-LO inhibitor, cinnamyl-3,4dihydroxy- $\alpha$-cyanocinnamate (CDC), are depicted in Figure 4A. Each was found to have an $\mathrm{IC}_{50}$ appropriate for inhibition of the 12LO (ETI, $20 \mu \mathrm{M}$; NDGA, $40 \mu \mathrm{M}$; CDC, $200 \mathrm{nM}$ ). These data strongly suggested that platelet $12-\mathrm{LO}$, as well as NADPH oxidase, were both required for $\mathrm{Ab}$-induced platelet fragmentation.

The direct effect of 12-LO products on platelet fragmentation was next tested in two different experimental settings. First,
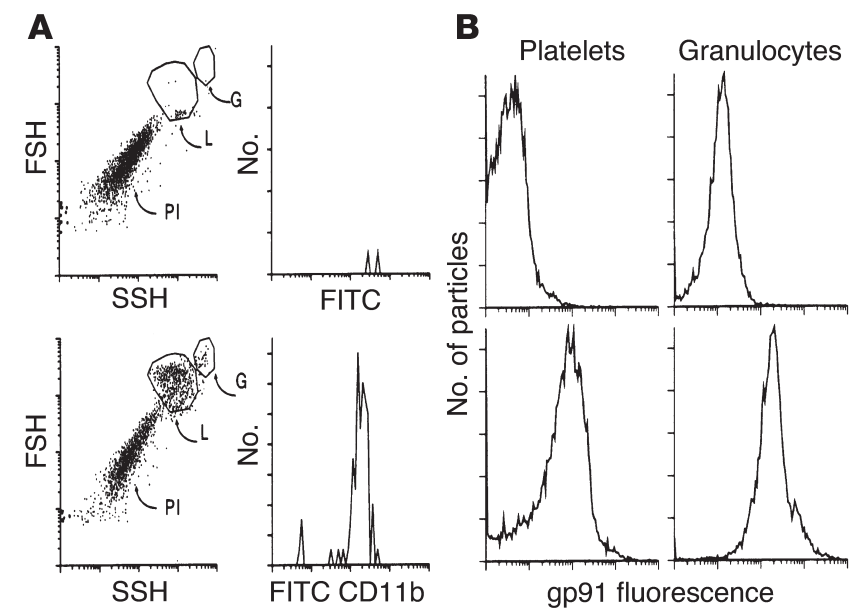

\section{Figure 1}

Absence of granulocytes and presence of the NADPH oxidase component gp $91^{\text {phox }}$ in gel-filtered platelets. (A) Fluorescence flow-cytometry comparison of a leukocyte/platelet preparation with gel-filtered platelets. Lower-left panel: forward scatter-side scatter plot of an enriched leukocyte/platelet preparation. G, granulocytes; L, lymphocytes; PI, platelets, as designated by their known scatter pattern. Upper-left panel: similar plot of gel-filtered platelets. Lower-right panel: anti-CD11b-FITC Ab reactivity specific for granulocytes/monocytes. Upper-right panel: similar plot of gel-filtered platelets. Granulocyte-reactive anti-CD11b-FITC $\mathrm{Ab}$ was unreactive with gel-filtered platelets $(<0.1 \%)$. $\mathrm{FSH}$, forward scatter height; SSH, side scatter height. (B) Reactivity of anti-gp91 Ab with granulocytes and platelets. Upper panels: control mouse mAb, reactivity with platelets and granulocytes. Lower panels: monoclonal anti-gp91 fluorescence. 

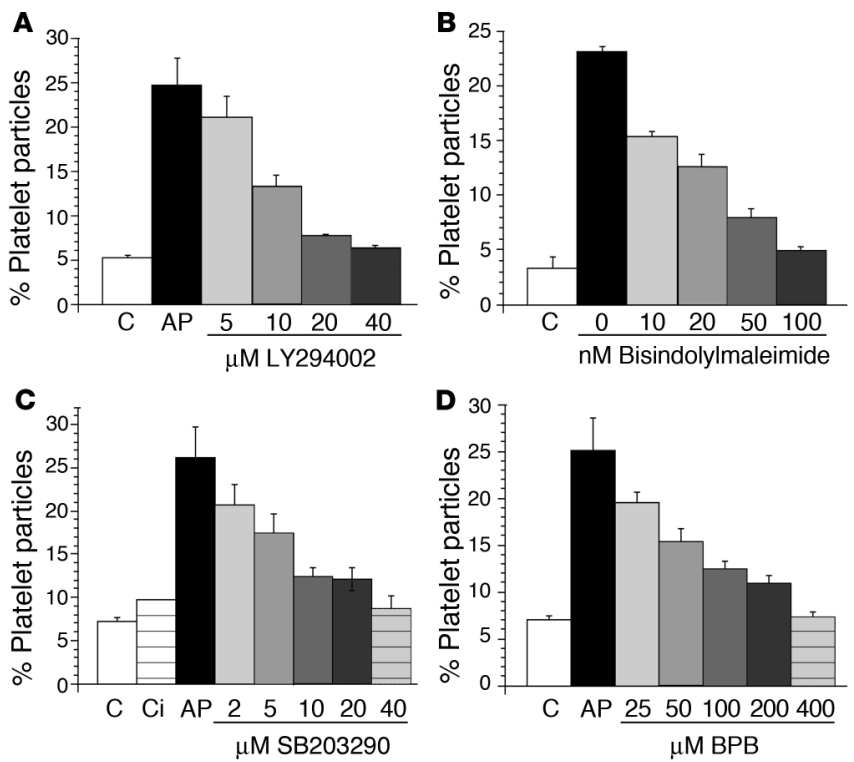

platelets were exposed to varying concentrations of $12(\mathrm{~S})$ HPETE and 12(S)-HETE, its stable breakdown product, and the level of platelet fragmentation was assessed. Both products were as effective as anti-GPIIIa49-66 Ab in inducing fragmentation (Figure 4B). In contrast, the nonbiologically derived stereoisomer, 12(R)-HETE, was without effect in this assay (Figure 4B), as was the granulocyte 5 -LO product, $\mathrm{LTB}_{4}$ (data not shown), indicating specificity. Second, confirming the connection between 12-LO and the NADPH oxidase system, we determined that, similar to Ab from HIV-1-ITP patients, 12(S)-HETE (200 nM) alone was sufficient to induce the translocation of $\mathrm{p} 47$ phox and $\mathrm{p} 67 \mathrm{phox}$ to platelet membranes (Figure 3D). As predicted from Figure 2, 12(S)-HETE-induced translocation was also inhibited by the protein kinase $C$ inhibitor, bisindolylmaleimide (Figure 3D). Furthermore, the time courses for platelet fragmentation induced by either patient anti-GPIIIa49-66 or by 12(S)-HETE were nearly identical (Figure 4C). Finally, confirming the central role of 12-LO in $\mathrm{Ab}$-induced platelet fragmentation, we quantified the generation of 12 (S)-HETE by platelets after exposure to $\mathrm{Ab}$. These platelets generate substantial levels of the biologically active lipid over a time course that is consistent with the induction of fragmentation by $\mathrm{Ab}$ (Figure 4D).

Platelet fragmentation with $12(\mathrm{~S})$-HETE requires interaction of platelet 12-LO with the platelet NADPH oxidase pathway. If 12(S)-HETE induces platelet fragmentation by ROS generation, it follows that interventions that inhibit ROS action should also block the 12(S)-HETE activity. Thus, we tested the ability of 12(S)-HETE $(100 \mathrm{nM})$ to fragment platelets in the presence of increasing concentrations of catalase and diphenylene iodonium (DPI). Both effectively blocked 12(S)-HETE-induced platelet fragmentation (Figure 5A) as they do Ab-induced platelet fragmentation (10). It also follows that $12(\mathrm{~S})$-HETE should induce the generation of measurable ROS, and, thus, we tested the ability of 12(S)-HETE treatment to induce platelet-dependent oxidation of the indicator dye, dichlorodihydrofluorescein (DCFH). 12(S)-HETE caused platelets to oxidize DCFH in a dose-dependent manner (Figure $5 \mathrm{~B})$. The concentration of $\mathrm{H}_{2} \mathrm{O}_{2}$ equivalents generated by platelets treated with 12(S)-HETE, as determined by DCFH oxidation, was
Figure 2

Inhibition of $A b$-induced platelet fragmentation with classic inhibitors of the NADPH oxidase pathway. Gel-filtered human platelets were preincubated with inhibitors at various concentrations for 15 minutes prior to the addition of $25 \mu \mathrm{g} / \mathrm{ml}$ affinity-purified anti-GPIIla49-66 (AP) or control (C) IgG for 4 hours. Ci, control IgG plus inhibitor; BPB, bomophenacyl bromide. Platelet fragmentation was monitored by flow cytometry of platelets labeled with anti-CD41-FITC Ab. Platelet fragments were monitored by enumeration of fluorescent particles in gates established to detect smaller particles. A, $n=4 ; \mathbf{B}, n=4 ; \mathbf{C}, n=3 ; \mathbf{D}, n=6$.

sufficient to induce platelet fragmentation when oxidation was induced with equivalent amounts of $\mathrm{H}_{2} \mathrm{O}_{2}(17 \mathrm{nM}, n=2)$. Similar observations were noted with $\mathrm{Ab}$ (data not shown).

The role of platelet 12-LO, Rac2, and NADPH oxidase in Abinduced platelet fragmentation was further demonstrated by the absence of Ab-induced thrombocytopenia in vivo in 12- $\mathrm{LO}^{-/}$, gp91 phox- $/-^{-}$, and Rac2 $2^{-/}$mice, as compared with WT mice (Figure $6 \mathrm{~A})$. Similar results were found in vitro where 12(S)-HETE was unable to induce platelet fragmentation in either gp91 phox-/- $^{-}$or $\mathrm{Rac}^{-/-}$mice (Figure 6B). Nevertheless, platelets from $12-\mathrm{LO}^{-/}$mice responded normally to $12(\mathrm{~S})$-HETE (Figure $7 \mathrm{~A}$ ), despite the absence of $\mathrm{Ab}$-induced platelet fragmentation. Ab-induced platelet
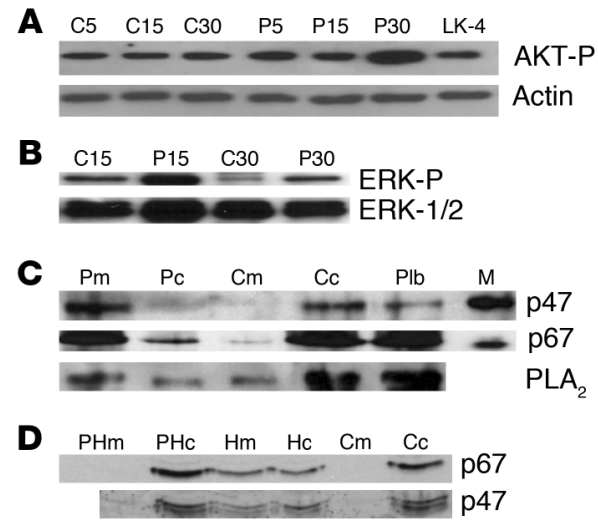

\section{Figure 3}

Functional evidence for NADPH oxidase activity. Gel-filtered human platelets were incubated with $25 \mu \mathrm{g} / \mathrm{ml}$ of control or patient $A b$ at $37^{\circ} \mathrm{C}$, extracted, and immunoblotted. (A) Effect of Ab on AKT activation. C5, $\mathrm{C} 15, \mathrm{C} 30$, and $\mathrm{P} 5, \mathrm{P} 15$, and $\mathrm{P} 30$ refer to minutes of incubation with control or patient $(P) A b$, respectively. LK-4 refers to incubation with irrelevant anti-GPIIla mAb. Actin is internal control (representative of five experiments). AKT-P, phosphorylated AKT. (B) Effect of patient Ab on ERK-1/2 activation. Upper lanes refer to reactivity with $A b$ directed against phosphorylated ERK (ERK-P). Lower lanes refer to Ab directed against internal control ERK protein (representative of three experiments). (C) Effect of Ab on translocation of p67 phox, p47 phox, and PLA ${ }_{2}$ from platelet cytosol to membrane. Platelets were incubated with $A b$ for 30 minutes at $37^{\circ} \mathrm{C}$, extracted, separated into cytosol and membrane components, and then immunoblotted with specific Ab's. M, marker for $\mathrm{p} 67^{\text {phox }}$ and $\mathrm{p} 47^{\text {phox }} \mathrm{Ab}$. PIt refers to platelets in buffer, prior to incubation. $\mathrm{Cc}, \mathrm{Cm}$ and $\mathrm{Pc}, \mathrm{Pm}$ refer to control and patient $\mathrm{Ab}$ directed against cytosol and membrane fractions, respectively. (D) Effect of 12(S)-HETE (200 nM) on translocation of p67phox and p47phox from platelet cytosol to membrane fractions. $\mathrm{Hm}, \mathrm{Hc}, \mathrm{Cm}$, and $\mathrm{Cc}$ refer to cytoplasmic and membrane fractions from 12(S)-HETE and control incubations, respectively. $\mathrm{PHm}$ and $\mathrm{PHc}$ refer to incubation with 12(S)HETE and protein kinase $\mathrm{C}$ inhibitor, bisindolylmaleimide at $20 \mathrm{nM}$. Representative of three experiments. 

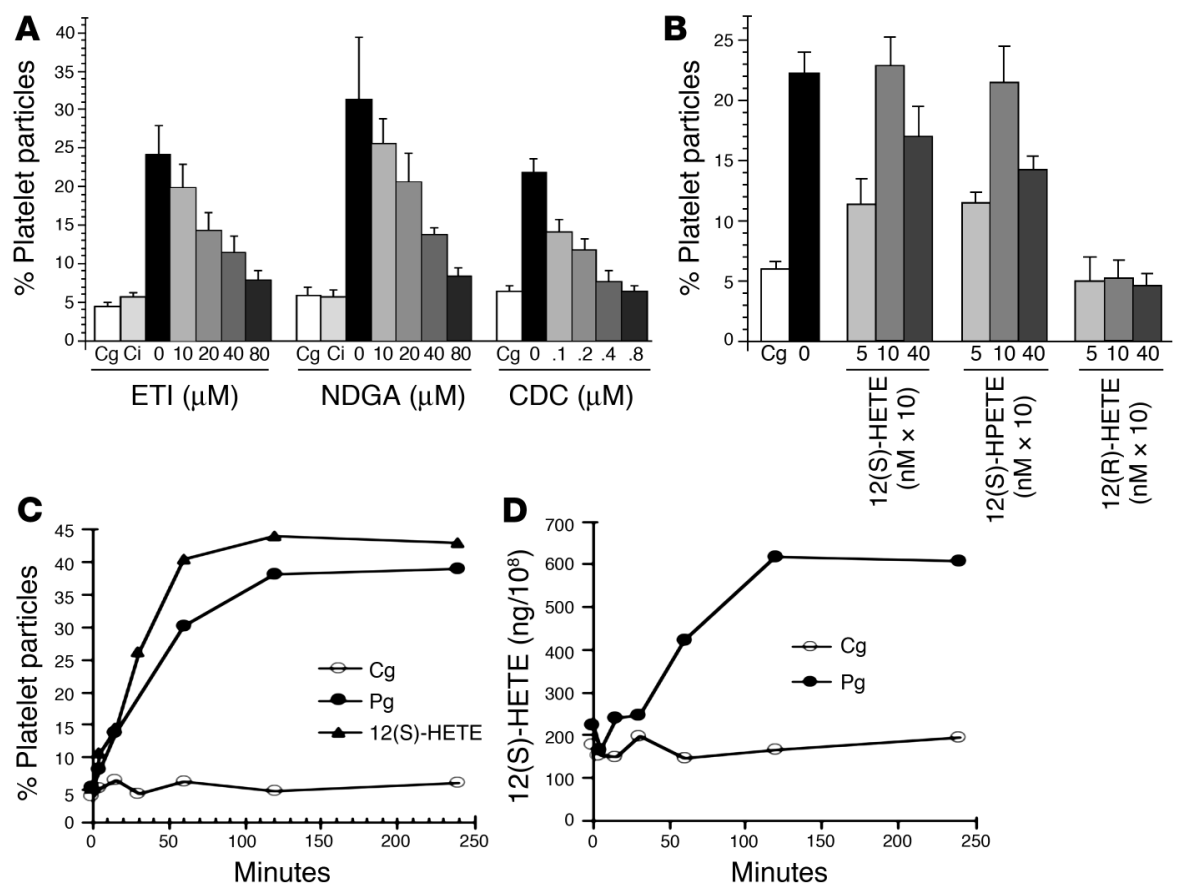

\section{Figure 4}

Role of platelet 12-LO in anti-GPIIla49-66induced platelet fragmentation. (A) Lipoxygenase inhibitors: 5-, 8-, 11-eicosatriynoic acid (ETI), nordihydroguairetic acid (NDGA) ( $n=4)$, and cinnamyl-3-, 4-dihydrocyanocinnamate (CDC) $(n=8)$ were added to gel-filtered human platelets 15 minutes prior to $A b$, and percentage of fragmentation measurements were made after a 4-hour incubation. $\mathrm{Cg}$, control lgG $(25 \mu \mathrm{g} / \mathrm{ml}) ; \mathrm{Ci}$, control IgG plus inhibitor $(25 \mu \mathrm{g} / \mathrm{ml}) ; 0$, patient IgG without inhibitor $(25 \mu \mathrm{g} / \mathrm{ml})$. (B) Effect of 12-LO products, 12(S)-HETE and $12(\mathrm{~S})$-HPETE $(n=8)$, on platelet fragmentation. 12(R)-HETE is a nonlipoxygenasederived stereosisomer $(n=3)$. (C) Comparison of $\mathrm{Ab}$ versus 12(S)-HETE-induced platelet fragmentation (typical of three experiments). (D) Effect of patient IgG (Pg) vs. control IgG (Cg) on platelet 12(S)-HETE production (typical of three experiments). fragmentation in 5- $\mathrm{LO}^{-/-}$mice was no different from WT mice (Figure $7 \mathrm{~B}$ ), indicating that the change in $12-\mathrm{LO}^{-/-}$was not a nonspecific effect related to the gene deletion.

\section{Discussion}

These data indicate that platelets have a functioning NADPH oxidase pathway capable of destroying platelets by the generation of ROS, following perturbation of platelet GPIIIa with a specific anti-GPIIIa49-66 Ab. Activation of NADPH oxidase requires prior activation of platelet 12-LO. It is therefore apparent that the 12-LO and NADPH oxidase pathways are linked, with 12-LO being upstream of NADPH oxidase. This is the first description of a role for 12-LO in platelets.

Suggestive evidence for the presence of an NADPH oxidase in platelets has been reported by us $(10)$, as well as others $(38,39)$. None of these reports present rigorous evidence for a functioning granulocyte-like NADPH oxidase system, however. Furthermore, the activation of the NADPH oxidase and subsequent platelet fragmentation induced by $\mathrm{Ab}$ requires generation of the 12-LO product, 12(S)-HETE. This article presents such evidence based upon the following observations: (a) gel-filtered platelets are free of contaminating granulocytes/monocytes; (b) platelets from two different NADPH oxidase-deficient mice, p47phox-/(10) and gp91phox-/-, failed to undergo either Ab or 12(S)HETE-induced platelet fragmentation, nor does the Ab induce thrombocytopenia in vivo; (c) human platelets contain membrane-bound gp919hox; (d) platelet p47phox, p67phox, and $\mathrm{CPLA}_{2}$ translocate from cytoplasm to membrane following Ab-induced fragmentation; (e) a diverse group of classic inhibitors of $\mathrm{NADPH}$ oxidase activation also inhibit $\mathrm{Ab}$-induced platelet fragmentation in vitro; (f) Ab-induced platelet fragmentation is associated with the activation of PI3K and ERK.

Indirect evidence for ligand-activated NADPH oxidase activity as well as components of the NADPH oxidase complex have been reported in nonphagocytic cells (smooth muscle) $(40,41)$, chondrocytes (42), kidney epithelium (43), and fibroblasts (44). Treatment with DPI, an inhibitor of the FAD component of neutrophil NADPH oxidase, can inhibit ROS production in these nonphagocytic cells when stimulated by ligands $(40,42,44)$. On the other hand, $\mathrm{p} 40^{\text {phox }}$ and $\mathrm{p} 47^{\mathrm{phox}}$ are dispensable for $\mathrm{NADPH}$ oxidase activity in a cell-free system, whereas p67phox is required (45). Indeed there has been little rigorous evidence supporting the existence of functional $\mathrm{p} 47^{\mathrm{phox}}$ and $\mathrm{p} 67^{\mathrm{phox}}$ in nonphagocytic cells. A recent suggestion has been made that other systems may regulate $\mathrm{p} 67^{\text {phox }}$ or that nonphagocytic cells do not require p67phox for ROS generation (46).

The role of ROS in cell messenger signaling is an emerging concept. Although high concentrations of ROS are used in host defense, there is evidence that lower concentrations (1-2\% of granulocyte generation) are required for cell signaling. There have been
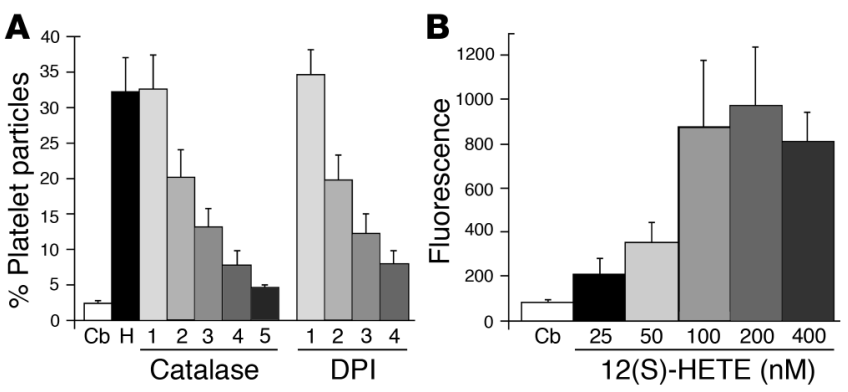

\section{Figure 5}

Effect of 12(S)-HETE on platelet oxidation. (A) Gel-filtered human platelets were preincubated with various concentrations of catalase and DPI for 15 minutes prior to the addition of 12(S)-HETE (100 nM) for 4 hours. $\mathrm{Cb}$, control buffer; $\mathrm{H}, 12(\mathrm{~S})$-HETE with no drug. Increasing numbers refer to doubling concentrations of catalase, starting at $125 \mu \mathrm{g} / \mathrm{ml}$, and DPI, starting at $10 \mu \mathrm{M}$. (B) Gel-filtered platelets were loaded with DCFH and incubated with 12(S)-HETE at various concentrations for 4 hours at $37^{\circ} \mathrm{C}$. Oxidation was measured by DCFH fluorescence. 

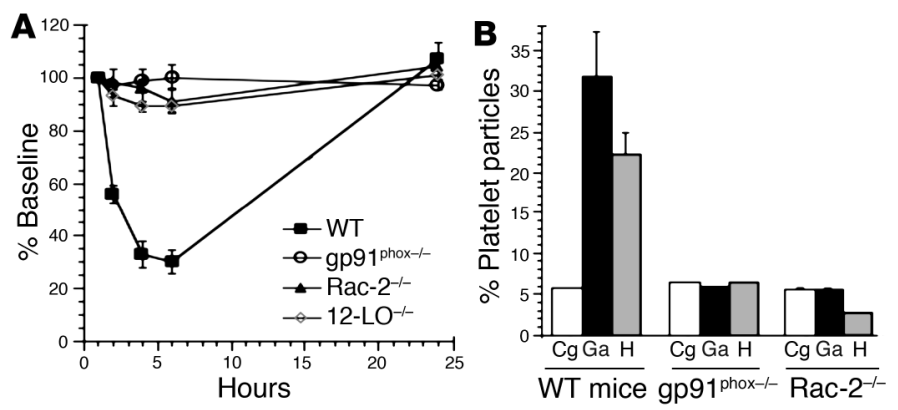

\section{Figure 6}

Effect of anti-GPIIla49-66 and 12(S)-HETE on platelet count and fragmentation of WT, NADPH oxidase gp91 phox---, Rac-2---, and 12$\mathrm{LO}^{-/-}$platelets. (A) Platelet count. Mice were treated with $25 \mu \mathrm{g}$ of control or patient anti-GPIIla49-66, and platelet counts were monitored at various time intervals ( $n=5$ for each group). (B) Fragmentation. Percentage of platelet particles were enumerated after in vitro exposure to anti-GPIIla49-66 (25 $\mu \mathrm{g} / \mathrm{ml})(\mathrm{Ga})$, control IgG $(\mathrm{Cg})$, or 12(S)-HETE (100 nM) (H) for 4 hours at $37^{\circ} \mathrm{C}$. several recent reports demonstrating a transient oxygen burst in cells stimulated by various cytokines: IL- $1 \beta$, TGF- $\beta 1$ (47), TNF- $\alpha$ $(42,48)$; growth factors: $\operatorname{EGF}(49,50), \operatorname{PDGF}(51,52), \operatorname{VEGF}(53)$, $\beta F G F(54)$, thrombin (55); and apoptotic signals (56-58). All induce ROS, which is required for tyrosine kinase receptor phosphorylation, cell stimulation, and mitogenesis, since ROS scavengers (and/or antioxidants) inhibit these reactions.

Of particular interest is the observation that the nonphagocytic cell cytokine and growth factor ROS pathways also require arachidonic acid release by $\mathrm{PLA}_{2}$. This requires the Rac-1 G-coupled protein (49), as well as activation of PI3K, ERK, and P38 MAPK, as is the case with the granulocyte NADPH oxidase system. ERK and p38 MAPK are involved in arachidonic acid release induced by $\mathrm{H}_{2} \mathrm{O}_{2}$ and PDGF in mesangial cells (34). ERK is known to increase $\mathrm{cPLA}_{2}$ activity following $\mathrm{CPLA}_{2}$ phosphorylation by ERK. The p38 MAPK also phosphorylates and activates $\mathrm{CPLA}_{2}$ in thrombin or collagen-activated platelets $(59,60)$. It has been reported in mesangial cells that both PDGF as well as $\mathrm{H}_{2} \mathrm{O}_{2}$ induce the phosphorylation/activation of ERK $(33,34,52)$. In human platelets, $\mathrm{H}_{2} \mathrm{O}_{2}$ induced phosphorylation/activation of $\mathrm{CPLA}_{2}$ with arachidonic acid release requires p38 MAPK (61).

The ROS burst of the cytokine/growth factor-induced pathways in nonphagocytic cells can be blocked by inhibitors of the lipoxygenase pathway. It is unlikely that lipoxygenase metabolism per se results in ROS generation (62), since arachidonic acid oxidation is independent of ROS production. It is more likely that 12-LO generates a downstream product that activates the NADPH oxidase pathway. In phagocytic cells, the pathway inhibited by lipoxygenase inhibitors can also be inhibited with DPI (antiflavoprotein oxidase), activated with $\mathrm{LTB}_{4}$, and blocked with antagonists of its receptor as well as by PTX (37). Yet, nonphagocytic cells do appear to express this NADPH oxidase pathway $(40,41,43,44)$. Thus, the presence, as well as relative roles of the various pathways, is unclear, and downstream events from receptor binding to induction of ROS are unknown.

Our current data provide an explanation for these observations by demonstrating that both the platelet 12 -LO and NADPH oxi- dase pathways are interconnected with the platelet $12-\mathrm{LO}$ pathway being upstream of the NADPH oxidase pathway. This is supported by our observations that the 12-LO product, 12(S)-HETE, induces platelet fragmentation even in platelets from 12-LO-deficient animals. That this is a specific pathway related to the generation of 12(S)-HETE is emphasized by the fact that Ab-induced platelet fragmentation is normal in platelets from 5-LO-deficient mice and by the observed inability of 12 (R)-HETE, a synthetic stereoisomer of the biologically active lipid, to induce platelet fragmentation. It is likely that both the 12-LO and NADPH ROSinducing pathways are involved in cell signaling, as with other nonphagocytic cells. In platelets, however, it can be pathophysiologically subverted to cell fragmentation and destruction following anti-GPIIIa49-66 ligation.

The physiological importance of the 12-LO and NADPH oxidase pathways in platelets has not yet been firmly established. Although platelets can engulf bacteria (63), there is no evidence that they can phagocytose and kill them. Platelets can generate ROS, particularly following activation with collagen, but not with ADP or thrombin; collagen-induced aggregation can be inhibited with catalase (64). It is suggested that Ab-induced ROS may have possible other effects on nonplatelet bystander cells such as megakaryocytes or endothelial cells, which could be contributing to the pathophysiology of the disease. Our data raise the possibility that 12-LO inhibition may be beneficial in reversing the thrombocytopenia in patients with AITP. Indeed, preliminary data reveal that this is so, since the platelet 12-LO inhibitor CDC can significantly inhibit in vivo platelet particle formation and thrombocytopenia in mice. Unfortunately, no drugs are currently available for use in humans, so further testing of this hypothesis will have to wait for the development of effective inhibitors.

\section{Methods}

Human population. Patient sera were obtained from early-onset HIV-1-infected thrombocytopenic patients without AIDS and control subjects (healthy laboratory personnel). These studies

\section{Figure 7}

Effect of anti-GPIIla49-66 and 12(S)-HETE on murine platelet fragmentation of (A) 12- $\mathrm{LO}^{-/-}$and (B) 5-LO-/- mice. Gel-filtered murine platelets were treated with control $\mathrm{Ab}(\mathrm{Ca})$, anti-GPIIla49-66 (Ga), 12(S)-HETE, or its steroisomer, 12(R)-HETE (rH). Note absence of anti-GPIIla49-66-induced platelet fragmentation with $12-\mathrm{LO}^{-/-}$mouse platelets and its presence with $5-\mathrm{LO}^{-/-}$mouse platelets. Note presence of $12(\mathrm{~S})$-HETE-induced platelet frag-

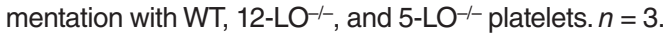
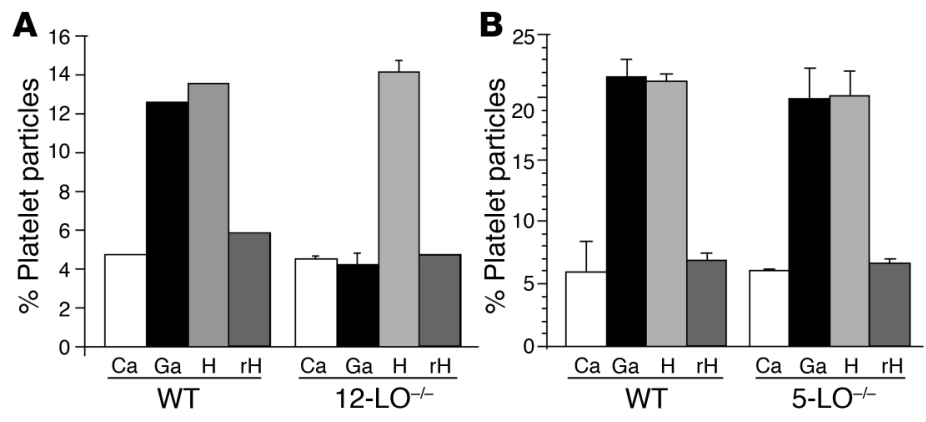
were approved by the New York University Medical Center Institutional Review Board.

Mice. WT mice were C57BL/6. All KO mice were of this back-

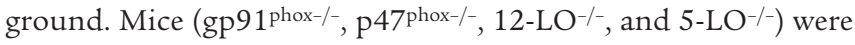
obtained from The Jackson Laboratory (Bar Harbor, Maine, USA). Rac-2-/- mice were kindly provided by David A. Williams (Indiana University School of Medicine, Indianapolis, Indiana, USA). Animal work was approved by the New York University School of Medicine Institutional Review Board.

Materials. All reagents were obtained from Sigma-Aldrich (St. Louis, Missouri, USA) unless otherwise designated. These reagents include inhibitors of G-coupled protein receptors, PTX; PI3K, LY294002; protein kinase C, bisindolylmaleimide; phospholipase $\mathrm{A}_{2}$, bromophenacyl bromide; ROS, catalase and DPI. The p38 MAPK inhibitor, SB203290, was obtained from Calbiochem-Novabiochem Corp. (San Diego, California, USA). Lipoxygenase inhibitors, ETI, NDGA, and CDC, and eicosanoids, 12(S)-HETE, 12(S)-HPETE, and 12(R)-HETE, were obtained from Biomol Research Laboratories (Plymouth Meeting, Pennsylvania, USA). DCFH diacetate (DCFH-DA) was obtained from Molecular Probes Inc. (Eugene, Oregon, USA). Goat polyclonal Ab's against $\mathrm{p} 47^{\mathrm{phox}}$ and $\mathrm{p} 67^{\mathrm{phox}}$ were obtained from Santa Cruz Biotechnology Inc., Santa Cruz, California, USA. Mouse mAb against $\mathrm{CPLA}_{2}$ was obtained from Upstate Biotechnology Inc. (Lake Placid, New York, USA). Mouse mAb against gp91 phox $^{\text {was }}$ a gift of H.L. Malech (NIH, Bethesda, Maryland, USA).

Affinity purification of antiplatelet GPIIIa49-66. IgG was isolated by polyethylene glycol-precipitable immunocomplexes (8), followed by protein A affinity chromatography, G-200 gel filtration, affinity chromatography and affinity purification on $2 \%$ paraformaldehyde-fixed platelets as described (7). The antiplatelet Ab was eluted with $0.1 \mathrm{M}$ glycine buffer, $\mathrm{pH} 2.9$, and further purified on a GPIIIa49-66 peptide-affinity column, as described (7).

Preparation of leukocyte/platelet-enriched suspension. Three milliliters of heparinized blood were added to $9 \mathrm{ml}$ of $1.25 \%$ dextran in isotonic saline, and red blood cells were allowed to settle to the bottom at room temperature for 90 minutes. The supernatant was first treated with 1 vol of distilled water for $60 \mathrm{sec}-$ onds to remove contaminating red blood cells, followed by $4 \mathrm{vol}$ of $4.25 \% \mathrm{NaCl}$ to achieve isotonicity. The supernatant was then centrifuged and the cell pellet washed and suspended in 1:5 volume of Tyrode's buffer.

Induction of platelet particles. Gel-filtered human platelets were prepared from blood collected in $5.2 \mathrm{mM}$ EDTA. Murine blood was collected by cardiac puncture into heparin ( $1 \mathrm{unit} / \mathrm{ml}$, final concentration). Gel-filtered platelets were prepared from platelet-rich plasma applied to a Sepharose $2 \mathrm{~B}$ column preincubated with Tyrode's buffer $(\mathrm{pH} 7.4)$. Platelets $\left(10^{8} / \mathrm{ml}\right)$ were either unlabeled or labeled with $10 \mu \mathrm{g} / \mathrm{ml}$ of an anti-CD61-FITC $\mathrm{mAb}$ for human or an anti-CD41b-FITC for murine GPIIb (PharMingen, San Diego, California, USA) for 30 minutes at $4^{\circ} \mathrm{C}$. The cells were then centrifuged at $1,000 \mathrm{~g}$ for 6 minutes at room temperature and resuspended in Tyrode's buffer. Ten microliters of platelet suspension $\left(10^{6}\right.$ platelets $)$ were then incubated with $10 \mu \mathrm{l}$ of anti-GPIIIa49-66 Ab $(250 \mu \mathrm{g} / \mathrm{ml})$ and $80 \mu \mathrm{l}$ of Tyrode's buffer for $0-4$ hours at $37^{\circ} \mathrm{C}$ and then stored in an ice bucket prior to measurements of the percentage of platelet particles by fluorescent flow cytometry.

Assay of platelet particle formation. Both fluorescent-labeled and unlabeled platelet particles were measured by flow cytometry, employing a FACScan (Becton Dickinson Immunocytometry Systems, Mountain View, California, USA). Similar results were obtained using both labeled and unlabeled platelets. Debris and dead cells were excluded using scatter gates. Only cells with low orthogonal light scattering were included in the sorting gates. Gates were adjusted for control platelets by exclusion of other blood cells. Fluorescent-labeled intact platelets were monitored in the right upper quadrant with the $y$ axis measuring forward scatter and the $x$ axis measuring fluorescence. For nonfluorescent cells, the $y$ axis measured forward scatter, and the $x$ axis measured side scatter. A shift in fluorescent or nonfluorescent particles from right upper quadrant to left upper quadrant and left lower quadrant reflected the percentage of platelet particle induction of 10,000 counted platelets/particles.

Assay of platelet oxidation. Gel-filtered platelets were loaded with 10 $\mu \mathrm{M}$ DCFH-DA for 30 minutes at $37^{\circ} \mathrm{C}$ as described previously (10) and challenged with platelet $\mathrm{Ab}$. Oxidation was quantified by measuring the increase in mean fluorescence with a flow cytometer.

Assay of PI3K and ERK-1/2 activity. Platelets $\left(5 \times 10^{7}\right)$ were incubated in $100 \mu \mathrm{l}$ of buffer containing control or patient IgG for 4 hours and then extracted in an equal volume of ice-cold platelet lysate buffer containing $50 \mathrm{mM}$ Tris, 5 mM EDTA, 1\% Triton X-100, $0.1 \%$ sodium cacodylate, $150 \mathrm{mM} \mathrm{NaCl}, 1 \mathrm{mM}$ PMSF, $1 \mathrm{mM}$ $\mathrm{Na}_{2} \mathrm{VO}_{3}, 1 \mathrm{mg} / \mathrm{ml}$ aprotinin, and $10 \mu \mathrm{g} / \mathrm{ml}$ leupeptin. Aliquots were then tested by immunoblot analysis.

PI3K phosphorylation was detected by phosphorylation of its substrate, AKT, by Western blot analysis using an affinity-purified polyclonal rabbit anti-pS475AKT Ab, followed by a donkey anti-rabbit IgG-HRP (Promega Corp., Madison, Wisconsin, USA), and developed by chemiluminescence with substrate provided by Pierce Chemical Co. (Rockford, Illinois, USA). Actin was run as an internal control with a goat polyclonal IgG (c-11; Santa Cruz Biotechnology Inc.), followed by a monoclonal anti-goat IgG-HRP (Sigma-Aldrich).

ERK-1/2 phosphorylation was detected with a mouse monoclonal IgG2a against pERK (E-4), followed by a goat anti-mouse IgG2a-HRP (Santa Cruz Biotechnology Inc.), and developed by chemiluminescence. Nonphosphorylated ERK was run as an internal standard with a rabbit polyclonal IgG against ERK-1 (K-23; Santa Cruz Biotechnology Inc.), followed by protein A-peroxidase (Sigma-Aldrich), and developed with chemiluminescence.

Translocation of p67phox, $p 47^{\text {phox }}$, and $c P L A_{2}$ to platelet membrane. Platelets $\left(5 \times 10^{7}\right)$ in $100 \mu \mathrm{l}$ of buffer containing control or patient IgG were incubated for 4 hours at $37^{\circ} \mathrm{C}$ and extracted in relaxation buffer, with membrane and cytosol fractions prepared as described (36), with minor modifications. Cells were suspended in $50 \mathrm{mM}$ HEPES (instead of $10 \mathrm{mM}$ PIPES) with protease inhibitors ( $1 \mathrm{mM}$ PMSF, $5 \mathrm{mM}$ EDTA, $10 \mu \mathrm{g} / \mathrm{ml}$ trayslol, and $10 \mu \mathrm{g} / \mathrm{ml}$ leupeptin or 1 tablet $/ 50 \mathrm{ml}$ of protease inhibitor cocktail, 1697498; Roche Diagnostics, Indianapolis, Indiana, USA) and frozen and thawed three times (instead of sonication). Insoluble cellular elements were removed by centrifugation at $30,000 \mathrm{~g}$ for 1 minute at $4^{\circ} \mathrm{C}$, and the supernatant was adjusted to $5 \mathrm{mM}$ EDTA, $1 \mathrm{mM} \mathrm{Na} \mathrm{VO}_{3}$, and $5 \mathrm{mM} \mathrm{NaF}$, as described. The supernatant was then centrifuged at $30,000 \mathrm{~g}$ for 1 hour and the membrane pellet was resuspended in the original suspension volume of $0.34 \mathrm{M}$ sucrose/half-strength relaxation buffer containing $1 \mathrm{mM}$ DTT. Cytosol and membranes were stored at $-70^{\circ} \mathrm{C}$. The $\mathrm{p} 47^{\mathrm{phox}}$, p $67^{\mathrm{phox}}$, and $\mathrm{CPLA}_{2}$ were then tested by immunoblot analysis with a specific $\mathrm{Ab}$, followed by an appro- 
priate secondary Ab linked to HRP (Santa Cruz Biotechnology Inc.). Blots were developed by chemiluminescence. Measurement of the cytosol marker, lactic dehydrogenase, revealed that the membrane fractions contained minimal trapping of total LDH $(9.5 \% \pm 1.4 \%, n=8)$ with an absence of significant variation before or after translocation.

Assay of platelet 12-HETE production. Gel-purified platelets $\left(10^{8} / 100\right.$ $\mu \mathrm{l})$ were incubated with control or patient $\mathrm{Ab}$ as described above. The interaction was stopped by addition of 1 vol of ice-cold acetone(giving a total volume of $200 \mu \mathrm{l}$ ) and the samples kept at $-70^{\circ} \mathrm{C}$ until extracted. Lipids were extracted into ether and analyzed by reverse-phase HPLC as previously described (65). The 12HETE was quantified by UV absorbance at $237 \mathrm{~nm}$.

Determination of mouse platelet counts. Twenty microliters of blood was drawn from the orbital sinus into unopettes (5854; Becton Dickinson Immunocytometry Systems ) with optimum anticoagulant concentration and diluent for platelet enumeration by phase-contrast microscopy.

1. Morris, L., Distenfeld, A., Amorosi, E., and Karpatkin, S. 1982. Autoimmune thrombocytopenic purpura in homosexual men. Ann. Intern. Med. 96:714-717.

2. Najean, Y., and Rain, J.-D. 1994. The mechanism of thrombocytopenia in patients with HIV. J. Lab. Clin. Med. 123:415-420.

3. Walsh, C., Nardi, M., and Karpatkin, S. 1984. On the mechanism of thrombocytopenic purpura in sexually-active homosexual men. N. Engl. J. Med. 311:635-639.

4. Savona, S., Nardi, M., and Karpatkin, S. 1985. Thrombocytopenic purpura in narcotics addicts. Ann. Intern. Med. 102:737-741.

5. Ratnoff, O., Menitove, J., Aster, R., and Lederman, M. 1983. Coincident classic hemophilia and "idiopathic" thrombocytopenic purpura in patients under treatment with concentrates of antihemophilic factor (factor VIII). N. Engl. J. Med. 308:439-442.

6. Karpatkin, S. 1997. Autoimmune (idiopathic) thrombocytopenic purpura. Lancet. 349:1531-1536.

7. Karpatkin, S., Nardi, M., and Hymes, K. 1995. Sequestration of anti-platelet GPIIIa antibody in rheumatoid factor-immune complexes of human immunodeficiency virus 1 thrombocytopenic patients. Proc. Natl. Acad. Sci. U. S. A. 92:2263-2267.

8. Nardi, M., and Karpatkin, S. 2000. Antiidiotype antibody against platelet anti-GPIIIa contributes to the regulation of thrombocytopenia in HIV-1-ITP patients. J. Exp. Med. 191:2093-2100.

9. Nardi, M., Liu, L.-X., and Karpatkin, S. 1997. GPIIIa (49-66) is a major pathophysiologically-relevant antigenic determinant for anti-platelet GPIIIa of HIV-1-related immunologic thrombocytopenia (HIV-1-ITP). Proc. Natl. Acad. Sci. U. S. A. 94:7589-7594.

10. Nardi, M., Tomlinson, S., Greco, M., and Karpatkin, S. 2001. Complement-independent, peroxide induced antibody lysis of platelets in HIV-1-related immune thrombocytopenia. Cell. 106:551-561.

11. Segal, B., Leto, T., Gallin, J., Malech, H., and Holland, S. 2000. Genetic, biochemical and clinical features of chronic granulomatous disease. Medicine. 79:170-200.

12. Wientjes, F., Segal, A., and Hartwig, J. 1997. Immunoelectron microscopy shows a clustered distribution of NADPH oxidase components in the human neutrophil plasma membrane. J. Leukoc. Biol. 61:303-312.

13. Someya, A., Nagaoka, I., and Yamashita, T. 1993. Purification of the $260 \mathrm{kDa}$ cytosolic complex involved in the superoxide production of guinea pig neutrophils. FEBS Lett. 330:215-218.

\section{Acknowledgments}

This work was supported by NIH grants HL-13336 and DA-04315 (to S. Karpatkin) and The Dorothy and Seymour Weinstein Research Fund. The authors thank Yelena Gor for her help in the 12-HETE assays.

Received for publication December 3, 2003, and accepted in revised form January 20, 2004.

Address correspondence to: Simon Karpatkin, Department of Medicine, New York University School of Medicine, 550 First Avenue, New York, New York 10016, USA. Phone: (212) 263-5609; Fax: (212) 263-0695; E-mail: simon.karpatkin@med.nyu.edu.

This work was presented at the Annual Meeting of the American Society of Hematology in Philadelphia, Pennsylvania, USA, on December 9, 2002. Portions of this work have appeared in abstract form (2002. Blood. 100:52A).
14. Lyer, S., Pearson, D., Nauseef, W., and Clark, R. 1994. Evidence for a readily dissociable complex of p47phox and p67phox in cytosol of unstimulated human neutrophils. J. Biol. Chem. 269:22405-22411.

15. Kobayashi, T., Robinson, J., and Seguchi, H. 1998. Identification of intracellular sites of superoxide production in stimulated neutrophils. J. Cell Sci. 111:81-91.

16. Abo, A., Webb, M., Grogan, A., and Segal, A. 1994. Activation of NADPH oxidase involves the dissociation of p21rac from its inhibitory GDP/GTP exchange protein (rhoGDI) followed by its translocation to the plasma membrane. Biochem. J. 298:585-591.

17. Diekmann, D., Abo, A., Johnston, C., Segal, A., and Hall, A. 1994. Interaction of Rac with p67phox and regulation of phagocytic NADPH oxidase activity. Science. 265:531-533.

18. Teahan, C., Rowe, P., Parker, P., Totty, N., and Segal, A. 1987. The X-linked chronic granulomatous disease gene codes for the beta-chain of cytochrome b245. Nature. 327:720-721.

19. Parkos, C., Allen, R., Cochrane, C., and Jesaitis, A. 1987. Purified cytochrome b from human granulocyte plasma membrane is comprised of two polypeptides with relative molecular weights of 91,000 and 22,000. J. Clin. Invest. 80:732-742.

20. Segal, A., et al. 1992. Cytochrome b-245 is a flavocytochrome containing FAD and the NADPHbinding site of the microbicidal oxidase of phagocytes. Biochem. J. 284:781-788.

21. Rotrosen, D., Yeung, C., Leto, T., Malech, H., and Kwong, C. 1992. Cytochrome b558: The flavinbinding component of the phagocyte NADPH oxidase. Science. 256:1459-1462.

22. Baggiolini, M., Boulay, F., Badwey, J., and Curnutte, J. 1993. Activation of neutrophil leukocytes: chemoattractant receptors and respiratory burst. FASEB J. 7:1004-1010.

23. Shiose, A., and Sumimoto, H. 2000. Arachidonic acid and phosphorylation synergistically induce a conformational change of $\mathrm{p} 47 \mathrm{phox}$ to activate the phagocyte NADPH oxidase. J. Biol. Chem. 275:13793-13801.

24. Qualliotine-Mann, D., Agwu, D., Ellenburg, M., McCall, C., and McPhail, L. 1993. Phosphatidic acid and diacylglycerol synergize in a cell-free system for activation of NADPH oxidase from human neutrophils. J. Biol. Chem. 268:23843-23849.

25. Gomez-Cambronero, J., and Keire, P. 1998. Phospholipase D: a novel major player in signal transduction. Cell. Signal. 10:387-397.

26. Wishart, M., Taylor, G., and Dixon, J. 2001. Phoxy lipids: revealing PX domains as phosphoinositide binding modules. Cell. 105:817-820.

27. Kanai, F., et al. 2001. The PX domains of p47phox and $\mathrm{p} 40$ phox bind to lipid products of PI(3)K. Nat. Cell Biol. 3:675-678.

28. Ellson, C.D., et al. 2001. PtdIns(3)P regulates the neutrophil oxidase complex by binding to the PX domain of p40phox. Nat. Cell Biol. 3:679-682.

29. Shiose, A., and Sumimoto, H. 2000. Arachidonic acid and phosphorylation synergistically induce a conformational change of $\mathrm{p} 47 \mathrm{phox}$ to activate the phagocyte NADPH oxidase. J. Biol. Chem. 275:13793-13801.

30. Inanami, O., et al. 1998. Activation of the leukocyte NADPH oxidase by phorbol ester requires the phosphorylation of $\mathrm{p}^{4} 7^{\mathrm{phox}}$ on serine 303 or 304. J. Biol. Chem. 273:9539-9543.

31. Faust, L.R., el Benna, J., Babior, B.M., and Chanock, S.J. 1995. The phosphorylation targets of 47 phox, a subunit of the respiratory burst oxidase. Functions of the individual target serines as evaluated by site-directed mutagenesis. J. Clin. Invest. 96:1499-1505.

32. Dana, R., Leto, T., Malech, H., and Levy, R. 1998. Essential requirement of cytosolic phospholipase A2 for activation of the phagocyte NADPH oxidase. J. Biol. Chem. 273:441-445.

33. Lin, L.-L., et al. 1993. cPLA2 is phosphorylated and activated by MAP Kinase. Cell. 72:269-278.

34. Hayama, M., Inque, R., Akiba, S., and Sato, T. 2002. ERK and P38 MAP kinase are involved in arachidonic acid release induced by $\mathrm{H} 202$ and PDGF in mesangial cells. Am. J. Physiol. Renal Physiol. 282:F485-F491.

35. Gijon, M., Spencer, D., Siddiqi, A., Bonventre, J., and Leslie, C. 2000. Cytosolic phospholipase A2 is required for macrophage arachidonic acid release by agonists that do and do not mobilize calcium. J. Biol. Chem. 275:20146-20156.

36. Dana, R., Malech, H., and Levy, R. 1994. The requirement for phospholipase $\mathrm{A} 2$ for activation of the assembled NADPH oxidase in human neutrophils. Biochem. J. 297:217-223.

37. Woo, C.-H., et al. 2002. Leukotrience B4 stimulates Rac-ERK cascade to generate reactive oxygen species that mediates chemotaxis. J. Biol. Chem. 277:8572-8578.

38. Seno, T., Nobutaka, I., Dayaun, G., and Masanori, O. 2001. Involvement of NADH/NADPH oxidase in human platelet ROS production. Thromb. Res. 103:399-409.

39. Krotz, F., et al. 2002. NAD(P)H oxidase-dependent platelet superoxide anion release increases platelet recruitment. Blood. 100:917-924.

40. Griendling, K., Minieri, C., Ollerenshaw, J., and 
Alexander, R. 1994. Angiotensin II stimulates $\mathrm{NADH}$ and NADPH oxidase activity in cultured vascular smooth muscle cells. Circulation. 74:1141-1148.

41. Bhunia, A., Han, H., Snowden, A., and Chatterjee, S. 1997. Redox-regulated signaling by lactosylceramide in the proliferation of human aortic smooth muscle cells. J. Biol. Chem. 272:15642-15649.

42. Lo, Y., and Cruz, T. 1995. Involvement of ROS in cytokine and growth factor induction of $\mathrm{c}$-fos expression in chondrocytes. J. Biol. Chem. 270:11727-11730

43. Cui, Z., and Douglas, J. 1997. Arachidonic acid activates c-jun $\mathrm{N}$-terminal kinase through NADPH oxidase in rabbit proximal tubular epithelial cells. Proc. Natl. Acad. Sci. U. S. A. 94:3773-3776.

44. Irani, K., et al. 1997. Mitogenic signaling mediated by oxidants in Ras-transformed fibroblasts. Science. 275:1649-1652.

45. Freeman, J., and Lambeth, J. 1996. NADPH oxidase activity is independent of $\mathrm{p} 47 \mathrm{phox}$ in vitro. J. Biol. Chem. 271:22578-22582.

46. Bokoch, G., and Diebold, B. 2002. Current molecular models for NADPH oxidase regulation by Rac GTPase. Blood. 100:2692-2696.

47. Thannickal, V.J., and Fanburg, B.L. 1995. Activation of an $\mathrm{H} 2 \mathrm{O} 2$-generating NADH oxidase in human lung fibroblasts by transforming growth factor beta 1. J. Biol. Chem. 270:30334-30338.

48. Woo, C.-H., et al. 2000. Tumor necrosis factor-alpha generates reactive oxygen species via a cytosolic phospholipase A2-linked cascade. J. Biol. Chem. 275:32357-32362.
49. Peppelenbosch, M., et al. 1995. Rac mediates growth factor-induced arachidonic acid release. Cell. 81:849-856.

50. Bae, Y., et al. 1997. Epidermal growth factor (EGF)induced generation of hydrogen peroxide. J. Biol. Chem. 272:217-221.

51. Sundaresan, M., Yu, Z., Ferrans, V., Irani, K., and Finkel, T. 1995. Requirement for generation of H202 for platelet-derived growth factor signal transduction. Science. 270:296-299.

52. Huwiler, A., Fabbro, D., and Pfeilschifter, J. 1995. Platelet-derived growth factor stimulates de novo synthesis of mitogen-activated protein kinase in renal mesangial cells. Eur. J. Biochem. 227:209-213.

53. Colavitti, R., et al. 2002. Reactive oxygen species as downstream mediators of angiogenic signaling by VEGF receptor-2/KDR. J. Biol. Chem. 277:3101-3108.

54. Lo, Y.Y., and Cruz, T.F. 1995. Involvement of reactive oxygen species in cytokine and growth factor induction of c-fos expression in chondrocytes. J. Biol. Chem. 270:11727-11730.

55. Patterson, C., et al. 1999. Stimulation of a vascular smooth muscle cell NAD(P)H oxidase by thrombin. J. Biol. Chem. 274:19814-19822.

56. Kane, D., et al. 1993. Bcl-2 inhibition of neural death: decreased generation of reactive oxygen species. Science. 262:1274-1277.

57. Hockenbery, D., Oltvai, Z., Yin, X.-M., Milliman, C., and Korsmeyer, S. 1993. Bcl-2 functions in an antioxidant pathway to prevent apoptosis. Cell. 75:241-251.

58. Buttke, T., and Sandstrom, P. 1994. Oxidative stress as a mediator of apoptosis. Immunol. Today. 15:7-10.

59. Kramer, R., et al. 1996. p38 mitogen-activated protein kinase phosphorylates cytosolic phospholipase A2 (cPLA2) in thrombin-stimulated platelets. J. Biol. Chem. 271:27723-27729.

60. Borsch-Haubolds, A., Kramer, R., and Watson, S. 1997. Phosphorylation and activation of cytosolic phospholipase A2 by $38-\mathrm{kDa}$ mitogen-activated protein kinase in collagen-stimulated human platelets. Eur. J. Biochem. 245:751-759.

61. Buschbeck, M., Ghomashchi, F., Gelb, M., Watson, S., and Borsch-Haubolds, A. 1999. Stress stimuli increase calcium-induced arachidonic acid release through phosphorylation of cytosolic phospholipase A2. Biochem. J. 344:359-366.

62. Feinmark, S., Uden, A., Palmblad, J., and Malmsten, C. 1983. Leukotriene biosynthesis by polymorphonuclear leukocytes from two patients with chronic granulomatous disease. J. Clin. Invest. 72:1839-1843.

63. Youssefian, A., Drouin, A., Masse, J.-M., Guichard, J., and Cramer, E. 2002. Host defense role of platelets: engulfment of HIV and Staphylococcus aureus occurs in a specific subcellular compartment and is enhanced by platelet activation. Blood. 99:4021-4029.

64. Pignatelli, P., Pulcinelli, F., Lenti, L., Gazzaniga, P., and Violi, F. 1998. Hydrogen peroxide is involved in collagen-induced platelet activation. Blood. 91:484-490.

65. Steel, D., Tieman, T., Schwartz, J., and Feinmark, S. 1997. Identification of 8-lipoxygenase pathway in nervous tissue of Aplysia californica. J. Biol. Chem. 272:18673-18681. 